\title{
Erratum: Evolution alters the consequences of invasions in experimental communities
}

Cara A. Faillace and Peter J. Morin

Nature Ecology \& Evolution 1, 0013 (2016); published 21 November 2016; corrected 6 March 2017.

In the version of this Article originally published there was a slight omission in Fig. 3 . The box plot for the $-\mathrm{I} /+\mathrm{R}$ treatment of $P$. bursaria should have been labelled $b$ to indicate its significant difference from the other treatments. This has been corrected in all versions of the Article. 